\title{
Five years follow-up of juvenile lupus nephritis: a single-center retrospective cohort study
}

\author{
Qijiao Wei, Wei Wang, Yanqing Dong, Linqing Zhong, Hongmei Song \\ Department of Pediatrics, Peking Union Medical College, Chinese Academy of Medical Sciences, Peking Union Medical College Hospital, Beijing, China \\ Contributions: (I) Conception and design: H Song; (II) Administrative support: None; (III) Provision of study materials or patients: None; (IV) \\ Collection and assembly of data: Q Wei, Y Dong, L Zhong; (V) Data analysis and interpretation: Q Wei, Y Dong, L Zhong; (VI) Manuscript writing: \\ All authors; (VII) Final approval of manuscript: All authors. \\ Correspondence to: Hongmei Song. Department of Pediatrics, Peking Union Medical College, Chinese Academy of Medical Sciences, Peking Union \\ Medical College Hospital, Shuaifuyuan NO.1, Beijing 100730, China. Email: songhm1021@hotmail.com.
}

\begin{abstract}
Background: We analyze the clinical manifestations and 5 years of follow-up outcomes of children with lupus nephritis $(\mathrm{LN})$ and provide a reference for clinicians.

Methods: The clinical data of children diagnosed with LN (n=62) from January 2012-2015 were collected and analyzed.

Results: The median age at the diagnosis was 12.0 years. The female to male ratio was $3.4: 1$. The most prevalent clinical features were mucocutaneous involvement and hematological involvement. Renal biopsy was performed on 38 patients. Class IV and class $\mathrm{V}$ were the most common findings. The lupus activity was improved markedly after 3 months treatment. The rate of survival was $98.3 \%$ in 5 years. The most common side effects of corticosteroid and other immunosuppressive agent drug treatment were corticosteroid-related hypertension and high intraocular pressure. The rate of cataracts, osteoporotic fracture, and visual field defects increased as the treatment progressed. Especially, the incidence of visual field defects in children is higher than adults.
\end{abstract}

Conclusions: The LN children showed a good prognosis. During the follow-up process, the adverse drug reactions, such as hormone-related hypertension and ocular hypertension, especially the visual field defects caused by hydroxychloroquine, cannot be excluded. However, multicenter long term follow-up studies are essential to substantiate the current data.

Keywords: Children; lupus nephritis; follow-up; treatment

Submitted Mar 27, 2021. Accepted for publication Jun 04, 2021.

doi: 10.21037/apm-21-720

View this article at: http://dx.doi.org/10.21037/apm-21-720

\section{Introduction}

Systemic lupus erythematosus (SLE) is an autoimmune disease characterized by immune inflammation. It involves multiple organs. Children with SLE are more serious than adults (1). The incidence of juvenile systemic lupus erythematosus (jSLE) is $0.4-0.6 / 100,000$. Previous studies have shown that up to $80 \%$ of jSLE patients develop lupus nephritis (LN) (2). Moreover, significant differences are detected in the characteristics and the severity of the disease among various ethnic groups (3). A few studies focused on the demographic characteristics, clinical manifestations, laboratory findings treatment outcomes, and follow-up of children with juvenile LN in China (4). A previous study on the research and recruitment of $\mathrm{LN}$ in China provides limited clinical guidance. Thus, the present study is a rare long-term follow-up study that describes the demographic, clinical features, and laboratory findings of 62 children with LN. A 5-year follow-up was conducted to provide a reference for clinicians. We present the following article in accordance with the STROBE reporting checklist (available at https://dx.doi.org/10.21037/apm-21-720). 


\section{Methods}

\section{Participants}

This study was approved by the Institutional Review Board of Peking Union Medical College Hospital (No. S-K885) and individual consent for this retrospective analysis was waived. The study was conducted in accordance with the Declaration of Helsinki (as revised in 2013). All the hospitalized children younger than 18 years old at the time of diagnosis between January 2012 and January 2015 were included in this retrospective study. Children with incomplete medical records at baseline were excluded from this study. The included children met the 1997 revised criteria of American College of Rheumatology (ACR) classification criteria of SLE and LN (5). The 2003 International Society of Nephrology/Renal Pathology Society (ISN/RPS) was used as a reference standard for the pathological classification of juvenile $\mathrm{LN}$ in the current study.

\section{Clinical data}

The demographic, clinical, and laboratory characteristics of the patients included in this study were collected retrospectively from outpatient medical records, hospitalized medical records, or telephone questionnaires at baseline, 3 months, 6 months, 1 year, 2 years, 3 years, 4 years, and 5 years. The clinical data included general parameters, such as gender, age of onset, type of renal pathology, and system of involvement. The laboratory measurements included white blood cell (WBC), platelet count (PLT), hemoglobin $(\mathrm{Hb})$, erythrocyte sedimentation rate (ESR), serum creatinine, urea nitrogen, 24-hour urinary protein (24h-UP), serum C3 and C4 levels, and autoantibodies such as antinuclear antibodies (ANA) and anti-doublestranded DNA (anti-dsDNA) levels, and systemic lupus erythematosus disease activity index (SLEDAI).

\section{Definitions}

Complete remission: The laboratory results, including blood cell count, renal function, albumin, serum Complement 3 (C3) and Complement 4 (C4), erythrocyte sedimentation rate (ESR), and $24 \mathrm{~h}-\mathrm{UP}<150 \mathrm{mg}$, were normal. Partial remission should meet all following criteria: (I) urinary protein decreased by $\geq 50 \%, 24 \mathrm{~h}-\mathrm{UP}<3,500 \mathrm{mg}$; (II) serum creatinine and urinary protein/creatinine improved by $50 \%$; (III) improved serum creatinine, urine protein/ creatinine $<1.0$, and all other laboratory tests are improved than the baseline. Remission rate $=$ complete remission (\%) + partial remission (\%). Active disease: Persistent low serum complement levels, persistent thrombocytopenia or anemia, deterioration of renal function, persistent or increase in proteinuria, or appearance of new manifestations (6-8). Recurrence: During the treatment, the SLEDAI is increased by $\geq 6$ during any $3-9$ months $(9,10)$. Lost to follow-up: children, who did not follow-up in the outpatient department or in the hospital, and it was confirmed repeatedly by phone that the child still could not be reached. Treatment adverse effects: the sepsis in this study included only the sepsis with positivity for peripheral blood culture. The mucocutaneous clinical features include rash, photosensitivity, alopecia, oral ulcers, and Raynaud's phenomenon, while the musculoskeletal manifestations are arthralgia or arthritis, myalgia or myositis (11-14).

\section{Statistical analysis}

The characteristics were summarized using descriptive statistics. The data were expressed as mean \pm standard deviation $(\bar{x} \pm s)$ for normally distributed continuous variables or median $(\mathrm{Q} 1, \mathrm{Q} 3)$ for continuous variables that do not conform to the normal distribution. The ratio is expressed as a percentage (\%). Herein, we used the Bland method (15) to compare the data in the literature. SPSS version 21.0 (SPSS Inc., Chicago, IL, USA) was used to compare the differences between the two groups using $t$-test or Mann-Whitney $\mathrm{U}$ test. The ratio was measured by $\chi^{2}$ test. $\mathrm{P}<0.05$ was considered statistically significant.

\section{Results}

\section{Demographic data and clinical characteristics at baseline}

A total of 62 patients, including 48 females and 14 males, were recruited in this study. The female to male ratio was 3.4:1. The median age at onset was 10.0 (range, 8.112.3 ) years. The median age at the diagnosis was 12.0 (range, 9.4-13.0) years. The time from onset to diagnosis was 0.3 (range, $0.1-2.0$ ) years. In the cohort, $8 / 62$ (12.9\%) patients had a family history of autoimmune disease.

This study showed that the mucocutaneous tissue (75.8\%) and blood (62.9\%) involved in the disease, while the incidence of neuropsychiatric, musculoskeletal, and cardiorespiratory involvement was $35.5 \%, 37.1 \%$, and 9.7\%, respectively (shown in Table 1). 
Table 1 Characteristics of the juvenile LN patients

\begin{tabular}{|c|c|}
\hline Parameter & $\mathrm{N}=62$ \\
\hline \multicolumn{2}{|l|}{ Demographic features } \\
\hline $\begin{array}{l}\text { Age at disease onset, years } \\
\text { (median, Q1, Q3) }\end{array}$ & $10.0(8.1,12.3)$ \\
\hline $\begin{array}{l}\text { Age at diagnosis, years } \\
\text { (median, Q1, Q3) }\end{array}$ & $12.0(9.4,13.0)$ \\
\hline $\begin{array}{l}\text { The duration from onset till } \\
\text { diagnosis (median, Q1, Q3) }\end{array}$ & $0.3(0.1,2.0)$ \\
\hline Sex (female/\%) & $48(77.4 \%)$ \\
\hline${ }^{\star}$ Family history of autoimmunity & $8(12.9 \%)$ \\
\hline \multicolumn{2}{|l|}{${ }^{\#}$ Clinical features } \\
\hline Mucocutaneous & $47(75.8 \%)$ \\
\hline Musculoskeletal & $23(37.1 \%)$ \\
\hline Hematological involvement & $39(62.9 \%)$ \\
\hline Neuropsychiatric involvement & $22(35.5 \%)$ \\
\hline Cardiorespiratory involvement & $6(9.7 \%)$ \\
\hline Kidney involvement & $62(100.0 \%)$ \\
\hline Patients with renal biopsy & $38(61.3 \%)$ \\
\hline I & $1(2.6 \%)$ \\
\hline II & $2(5.3 \%)$ \\
\hline III & $6(15.8 \%)$ \\
\hline IV & $16(42.1 \%)$ \\
\hline V & $7(18.4 \%)$ \\
\hline VI & $0(0.0 \%)$ \\
\hline $\mathrm{III+V}$ & $1(2.6 \%)$ \\
\hline $\mathrm{IV}+\mathrm{V}$ & $5(13.6 \%)$ \\
\hline
\end{tabular}

Q1, first quartile; Q3, third quartile. *, family history of autoimmunity included type 1 diabetes, SLE, thyroid disease, rheumatoid arthritis and connective tissue disease (7). ", the clinical feature of mucocutaneous include rash, photosensitivity, alopecia, oral ulcers, and Raynaud's phenomenon. Musculoskeletal manifestations are arthralgia or arthritis, myalgia or myositis (15).

\section{Results of renal biopsy}

Among the $62 \mathrm{LN}$ children, 38 (61.3\%) underwent renal biopsy, while the remaining 24 patients without renal biopsy were unable to cooperate due to their young age or had contraindications or their parents were concerned about the risk. Class IV and $\mathrm{V}$ were the most common findings on renal biopsy, and the ratio was $42.1 \%$ and $18.4 \%$, respectively. The ratio of Class III and $\mathrm{IV}+\mathrm{V}$ was $15.8 \%$ and $13.6 \%$, respectively. The patients in this study had fewer type I, II, and III+V stages, while no class VI was detected in our patients (shown in Table 1).

\section{Laboratory findings}

Clinical data from 62, 48, 45, 47, 43, 43, 38, 29 cases of children were collected at the time of onset, 3 months, 6 months, 12 months, 2 years, 3 years, 4 years, and 5 years, respectively, while the data were not collected from 2 patients lost to follow-up. The number of telephone follow-up cases at each time point were $0(0 \%), 14$ (22.6\%), 15 (25.0\%), 13 (21.6\%), 17 (28.3\%), 17 (28.3\%), $22(36.7 \%)$, and $31(51.7 \%)$, respectively.

The laboratory data for WBC, PLT, Hb, 24h-UP, and ESR are listed in Table 2. The positive rates of ANA and anti-dsDNA antibodies were $100.0 \%$ and $70.8 \%$, respectively. The low $\mathrm{C} 3$ and $\mathrm{C} 4$ rates were $83.9 \%$ and $77.4 \%$, respectively. The positive rates of anti-Sm antibody, anti-SSA antibody, and anti-SSB antibody were $27.4 \%$, $43.5 \%$, and $16.1 \%$, respectively. All children were not tested for antiphospholipid antibodies. The positive rates of anticardiolipin antibody (ACL), anti- $\beta 2$ glycoprotein 1 antibody ( $\beta 2 \mathrm{GP} 1)$, and lupus anticoagulant (LA) were 7.4\%, 3.7\%, and $22.2 \%$, respectively.

After 3 months of treatment, $\mathrm{WBC}, \mathrm{Hb}$, and PLT were higher than that before the treatment. PLT and $\mathrm{Hb}$ were increased at 6 months as compared to that at 3 months, while WBC was decreased slightly, and the $24 \mathrm{~h}-\mathrm{UP}$ decreased significantly at 3 and 6 months. Urea nitrogen and ESR were reduced significantly compared to the baseline, and the rates of low $\mathrm{C} 3$ and $\mathrm{C} 4$ and anti-dsDNA antibody decreased significantly at 3 months (shown in Table 3).

\section{Medication data}

All the patients received oral corticosteroid treatment $(n=62$, $100 \%$ ), and in the majority of the patients, it was combined with hydroxychloroquine (HCQ) $(n=52,83.9 \%)$. The frequency of pulse corticosteroid and cyclophosphamide was $72.6 \%(n=45)$ and $75.8 \%(n=47)$, respectively. Mycophenolate mofetil (MMF) was the most frequently used agent for lupus nephritis $(n=42,67.7 \%)$, followed by cyclosporine $(\mathrm{n}=11,17.7 \%)$. Three $(4.8 \%)$ patients in the cohort used tacrolimus, while leflunomide was used in $7(11.3 \%)$ children. One patient was treated with methotrexate because of chronic arthritis, and 6 recurrent 
Table 2 Laboratory findings of juvenile LN at baseline

\begin{tabular}{|c|c|}
\hline Parameter & At baseline $(\mathrm{N}=62)$ \\
\hline WBC (×10/L) (median, Q1, Q3) & $6.5(2.8,8.9)$ \\
\hline Hemoglobin (g/L) & $102.4 \pm 19.8$ \\
\hline Platelet (×109/L) (median, Q1, Q3) & $168[94,267]$ \\
\hline 24-h urinary proteins (g/day) (median, Q1, Q3) & $1.7(0.9,6.1)$ \\
\hline Serum creatinine (mmol/L) (median, Q1, Q3) & $57.0(48.0,79.0)$ \\
\hline Blood urea nitrogen (mmol/L) (median, Q1, Q3) & $7.5(4.9,11.3)$ \\
\hline $\begin{array}{l}\text { Erythrocyte sedimentation rate }(\mathrm{mm} / \mathrm{h}) \\
\text { (median, Q1, Q3) }\end{array}$ & $36[21,73]$ \\
\hline Low C3 level, n (\%) & $52(83.9)$ \\
\hline Low C4 level, n (\%) & $48(77.4)$ \\
\hline Antinuclear antibody positivity, n (\%) & $62(100.0)$ \\
\hline Anti-dsDNA positivity, n (\%) & $44(70.8)$ \\
\hline Anticardiolipin antibody positivity, n (\%) (N=54) & $4(7.4)$ \\
\hline Anti $\beta 2$ glycoprotein 1 positivity, $n$ (\%) (N=54) & $2(3.7)$ \\
\hline Lupus anticoagulant positivity, n (\%) (N=54) & $12(22.2)$ \\
\hline Anti-Sm antibody positivity, $\mathrm{n}(\%)$ & $17(27.4)$ \\
\hline Anti-SSA antibody positivity, n (\%) & $27(43.5)$ \\
\hline Anti-SSB antibody positivity, n (\%) & $10(16.1)$ \\
\hline
\end{tabular}

$\mathrm{N}, \mathrm{n}$, number; Q1, first quartile; Q3, third quartile; WBC, white blood cell; C3, Complement 3; C4, Complement 4; Anti-dsDNA, anti-double stranded DNA.

patients were treated with rituximab, while one was treated with the JAK-STAT signaling pathway-targeted drug tofacitinib (shown in Table 4).

\section{Follow-up outcome}

After 3 months of treatment, 49/62 children were relieved, and the remission rate was $79.0 \%$. At 6 months, 2 patients were lost to follow-up, 53 were relieved, 6 retained the active disease, and 1 patient died of renal failure at 5 months. The remission rate was $88.3 \%$, and the mortality rate was $1.7 \%$. After 1 year of treatment, 58 patients were relieved, 1 patient was still in the active state, and 1 patient showed a relapse; the remission rate was $96.7 \%$, and the recurrence rate was $10.2 \%$. The follow-up data are presented in Table 3. At 5 years, 4 patients discontinued medical treatment, 20 patients relapsed, and 1 patient died; the recurrence rate was $33.3 \%$, and the mortality rate was $1.7 \%$.

\section{Treatment of adverse effects}

The most common side effects of drug treatment were corticosteroid-related hypertension $(54.8 \%)$ and corticosteroid-related high intraocular pressure $(38.7 \%)$ at 3 months. Four patients had visual field defects (6.5\%) due to hydroxychloroquine, 2 patients had sepsis $(3.2 \%)$, and 3 patients had cataracts (4.8\%). After 1 year of treatment, one child presented osteoporotic fracture, and the occurrence ratio was $1.7 \%$. All the follow-up data are presented in Table 3. At 5 years, $65.0 \%$ of the children exhibited corticosteroid-related hypertension, $53.3 \%$ of the patients had high intraocular pressure, and $5.0 \%$ of the patients had sepsis. Moreover, $16.7 \%$ of the patients had cataract, and $16.7 \%$ had visual field defects. About half of the children developed corticosteroid-related hypertension and high intraocular pressure after ingesting glucocorticoid. The rate of cataract, osteoporotic fracture, and visual field defects was increased with the treatment progress.

\section{Discussion}

The gender of the LN children in the current study is consistent with the LN cohort study in Japanese and UK children $(6,16)$, and the proportion of female children is about $80 \%$. The median age of diagnosis was 12 -years-old. The studies in the UK and Japan reported that the average age at the time of diagnosis was 12.3 and 13 years old. Based on the data reported in the literature, we use the Bland method (15) to compare the two datasets and did not find any significant difference in these cohorts. The time from the onset to diagnosis is $0.3(0.1-2.0)$ years, which is consistent with the results of the study on jSLE from Turkey $(14,17)$. About $12.9 \%$ of the children in the current study have a family history of autoimmune diseases. Thus, it was shown that about $10 \%$ of SLE patients have a family history, which was consistent with the current findings (18).

In our LN cohort, mucocutaneous and blood were involved, and $35.5 \%$ of the children had neuropsychiatric symptoms. This higher incidence as compared to that in the UK could be related to the high incidence of the nervous system affecting the Asian population $(19,20)$. Reportedly, the incidence of neuropsychiatric involvement was $7.7-45 \%$. Also, the inconsistent diagnostic criteria for neuropsychiatric lupus is a major cause of the difference $(21,22)$. In this study, musculoskeletal involvement was detected in 23/62 (37.1\%) children. In addition, arthritis is common in SLE and is one of the diagnostic criteria. Unlike juvenile idiopathic 
Table 3 Five-year follow-up data of juvenile LN

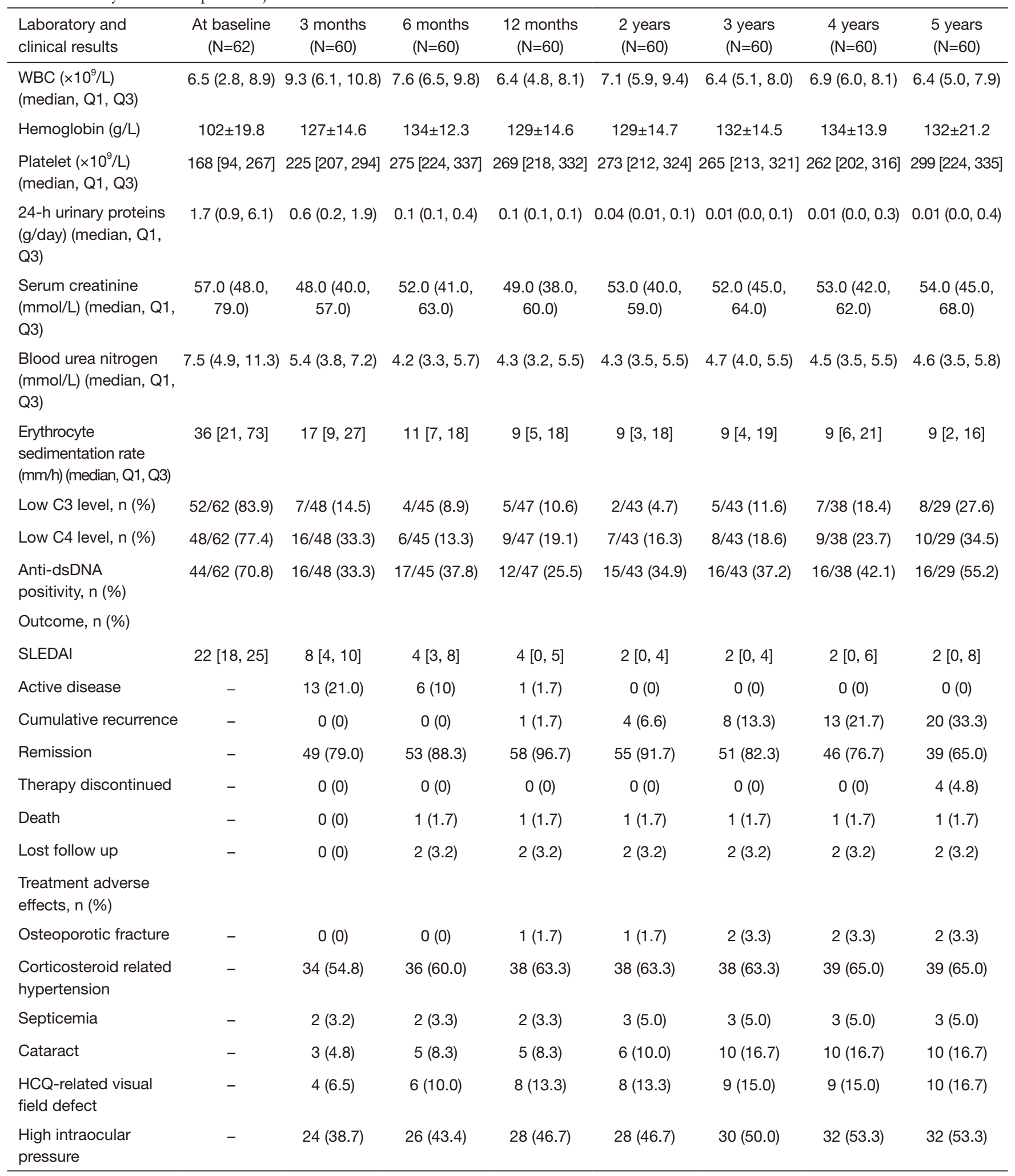

N, number; Q1, first quartile; Q3, third quartile; WBC, white blood cell; C3, complement 3; C4, complement 4; anti-dsDNA, anti-double stranded DNA; SLEDAI, systemic lupus erythematosus disease activity index; $\mathrm{HCQ}$, hydroxychloroquine. 
Table 4 Medication data of juvenile LN patients

\begin{tabular}{|c|c|c|c|}
\hline Medication & \multicolumn{2}{|c|}{ Initial treatment $(\mathrm{n}=62), \mathrm{n}(\%)$} & $\begin{array}{l}\text { Induced therapy after relapse } \\
\qquad(\mathrm{n}=20), \mathrm{n}(\%)\end{array}$ \\
\hline Corticosteroid & $62(100.0)$ & $62(100.0)$ & $20(100.0)$ \\
\hline Oral corticosteroid & $17(27.4)$ & $62(100.0)$ & $14(70.0)$ \\
\hline Methylprednisolone pulse therapy & $45(72.6)$ & $0(0.0)$ & $6(30.0)$ \\
\hline Mycophenolate mofetil & $7(11.3)$ & $42(67.7)$ & $6(30.0)$ \\
\hline Cyclosporine & $3(4.8)$ & $11(17.7)$ & $3(15.0)$ \\
\hline Tacrolimus & $1(1.6)$ & $3(4.8)$ & $2(10.0)$ \\
\hline Hydroxychloroquine & $52(83.9)$ & $46(74.2)$ & - \\
\hline Rituximab & $0(0.0)$ & $0(0.0)$ & $6(30.0)$ \\
\hline Tofacitinib & $0(0.0)$ & $0(0.0)$ & $1(5.0)$ \\
\hline
\end{tabular}

arthritis, SLE arthritis is non-invasive $14 \%$ incidence in Asian children (23). Another study reported that the incidence of arthritis in SLE could be about 85.7\% (24), and most of the cases were acute arthritis. Conversely, chronic arthritis is rare in SLE children, with the prevalence is $2.6 \%(25)$. In this study, 1 patient had chronic arthritis that was treated with methotrexate. Cardiopulmonary involvement in children with $\mathrm{LN}$ accounted for $9.7 \%$ of the cases, mainly including interstitial pneumonia and myocarditis, while pericardial effusion was not included in the scope of cardiac involvement. The proportion of these systems was consistent with that reported previously $(24,26)$. The above clinical manifestations suggested that the clinical manifestations are diverse in different races and populations. These differences were closely related to genetics, environment, and sample size.

The rate of renal biopsy in this study was $61.3 \%$. Many children had clear renal biopsy contraindications in our study. Some children had been treated without renal biopsy in other hospitals. Concurrently, parents were concerned about the risk of renal biopsy and refused it. The criteria for renal biopsy in our institute are as follows: (I) $24 \mathrm{~h}-\mathrm{UP}$ $>150 \mathrm{mg}$; (II) have not been treated by using glucocorticoids and immunosuppressants; (III) usually, the age of children is more than 8 years old, and they can follow doctors' instructions, because our institute has not carried out renal biopsy technique for children under general anesthesia; (IV)
The children and their parents agree to renal biopsy. These are the reasons for the low renal penetration rate in this study. Class IV and V LN were the most common findings, which were consistent with the literature reports.

ANA and anti-dsDNA antibody were detected in 62 patients, with positive rates of $100.0 \%$ and $70.8 \%$, respectively. This phenomenon was consistent with the positive rate of jSLE reported by another center in China. The positive rates of ANA and anti-dsDNA antibody were $97.62 \%$ and $70 \%$, respectively (27). In this study, the proportion of low complement $\mathrm{C} 3$ and $\mathrm{C} 4$ was $83.9 \%$ and $77.4 \%$, respectively. The antibodies with high positive rates also included anti-SSA antibodies (43.5\%) and antiSm antibodies (27.4\%). The cohort consisted of 28 patients with positive SSA antibodies and/or SSB antibodies, and 10 patients were diagnosed with secondary Sjögren's syndrome. Next, 54/62 children were tested for antiphospholipid antibodies. The rate of ACL-positive was $7.4 \%, \beta 2 \mathrm{GP} 1$-positive was $3.7 \%$, and LA-positive was $22.2 \%$. Of the 14 patients, who were positive for antiphospholipid antibody, 1 had abnormal coagulopathy, and 1 had cerebral infarction. During the follow-up period, arteriovenous thrombosis was not detected in the other children.

At the baseline, blood tests of children indicated a decrease in WBCs or Hb or PLT because of the hematological involvement. Children usually had increased 
ESR and elevated urinary protein. After treatment, the laboratory test results showed a gradual return to the normal at 3 months.

All patients in our center received oral corticosteroid treatment, and $83.9 \%$ of them received corticosteroid combined with HCQ. For patients with pathological renal Class I and II without neuropsychiatric involvement, prednisone, and MMF were administered. Intravenous infusion of methylprednisolone and cyclophosphamide for children with Class III and IV were used as induction treatment, and MMF was used as the maintenance treatment after cyclophosphamide. We usually use pulse corticosteroid and cyclophosphamide for induction therapy. Intravenous injection of methylprednisolone $15-30 \mathrm{mg} /(\mathrm{kg} \cdot \mathrm{d})$, the maximum amount of $500 \mathrm{mg} / \mathrm{d}$, for 3 days, once a week, continuous use for 2 weeks and intravenous cyclophosphamide pulse therapy every 2 weeks, every time continuous 2 days, $8-12 \mathrm{mg} /(\mathrm{kg} \cdot \mathrm{d})$, for 3 months, the maximum daily dose is $1 \mathrm{~g}$. And changed to monthly cyclophosphamide pulse treatment for another 3 months. The maintenance treatment is followed by sequential MMF [20-30 mg/(kg.d)]. Oral prednisone for 6 to 8 weeks, gradually reduce the dose according to the therapeutic effect, until it reaches a maintenance dose of $5-10 \mathrm{mg} / \mathrm{d}$. In the case of children with Class $\mathrm{V}$, cyclosporine or tacrolimus was used. Other immunosuppressive agents, such as rituximab, were often used for relapse treatment. In the present study, one child showed active disease after one recurrence; importantly, rituximab and other immunosuppressive agents could not control his proteinuria, and hence, tofacitinib, a JAK inhibitor, was administered for the treatment. Some studies have shown that the JAK/STAT pathway is essential for resisting infection and maintaining immune tolerance (28). Several studies have shown that this signaling pathway is involved in the pathogenesis of SLE and tofacitinib reduces proteinuria in mice SLE model and improves their survival rate (29). However, the child developed severe sepsis after using the drug, and proteinuria had not been controlled effectively. Currently, the child is treated with cyclophosphamide, albeit the proteinuria is not yet relieved.

After treatment, the remission rate reached $79.0 \%$ at 3 months and increased at 6 months. The majority of children could achieve remission at 1 year. One patient had recurrence at 1 year, which increased over time. After 5 years of follow-up, the recurrence rate was noted to be $33.3 \%$. Some patients relapsed more than one time. Although no obvious cause of recurrence was identified, it could be ascribed to infection and sun exposure. One patient died of renal failure at 5 months after the onset. The current study showed that the survival rate at 5 years was $98.3 \%$. Another study reported that the 3- and 5-year survival rates of children with $\mathrm{LN}$ are $93.59 \%$ and $87.80 \%$, respectively (4).

Glucocorticoids and immunosuppressant effectuate adverse effects while relieving the disease. This study showed that about half of the children had hypertension or high intraocular pressure after taking glucocorticoids. With prolonged medication, some children exhibited osteoporosis and also fragility-related fractures. Two patients developed spinal compression fractures at 3 years post-drug treatment. At the same time, the incidence of cataracts increased with the prolonged period of administering glucocorticoids. At 5 years follow-up, $16.7 \%$ of the children had cataracts. This study showed that $5.0 \%$ of the children developed severe sepsis during treatment. A single-center cohort study found that 35/87 patients with SLE had at least one infection, and one of the risk factors associated with infection was oral or intravenous glucocorticoids and intravenous cyclophosphamide (30). HCQ is safe and effective for SLE patients. About $83.9 \%$ of the children were treated with HCQ in this study. However, the main adverse effect was retinopathy. In addition, visual field defects might occur after 3 months of HCQ treatment. As the treatment progresses, the incidence of visual field defects increased gradually, and the incidence of visual field defects was $16.7 \%$ at 5 years. A systematic review found that the incidence of retinopathy in patients treated with HCQ for about 10 years was only $0.1 \%(31)$. In the present study, the high incidence of visual field defects might be related to the young age. China's Rheumatology Association recommends that patients with high-risk factors for retinopathy should undergo an eye exam once a year during the treatment (32). In order to avoid the adverse consequences, we recommend that children who receive HCQ have an eye exam at least half a year.

This study describes the demographic data, clinical features, and laboratory findings of 62 children with LN. A 5-year follow-up provided a reference for the clinicians. However, there are still deficiencies in this study. This was a single-center retrospective clinical study, and the loss to follow-up may have some bias. Therefore, a multicenter, long-term follow-up study is essential to obtain reliable data.

\section{Acknowledgments}

Thanks go to Wei Min for valuable discussion and to 
Li Ji, Wang Changyan and Yu Zhongxun for providing with literature knowledge and information in this paper.

Funding: This study was funded by CAMS Innovation Fund for Medical Sciences (CIFMS) (2016-I2M-1-008) and Beijing Science and Technology Association Jinqiao Engineering Seed Fund C Project (JQ17032).

\section{Footnote}

Reporting Checklist: The authors have completed the STROBE reporting checklist. Available at https://dx.doi. org/10.21037/apm-21-720

Data Sharing Statement: Available at https://dx.doi. org/10.21037/apm-21-720

Conflicts of Interest: All authors have completed the ICMJE uniform disclosure form (available at https://dx.doi. org/10.21037/apm-21-720) and reports receiving funding from CAMS Innovation Fund for Medical Sciences (CIFMS) (2016-I2M-1-008) and Beijing Science and Technology Association Jinqiao Engineering Seed Fund C Project (JQ17032). The other authors have no conflicts of interest to declare.

Ethical Statement: The authors are accountable for all aspects of the work in ensuring that questions related to the accuracy or integrity of any part of the work are appropriately investigated and resolved. This study was approved by the Institutional Review Board of Peking Union Medical College Hospital (No. S-K885) and individual consent for this retrospective analysis was waived. The study was conducted in accordance with the Declaration of Helsinki (as revised in 2013).

Open Access Statement: This is an Open Access article distributed in accordance with the Creative Commons Attribution-NonCommercial-NoDerivs 4.0 International License (CC BY-NC-ND 4.0), which permits the noncommercial replication and distribution of the article with the strict proviso that no changes or edits are made and the original work is properly cited (including links to both the formal publication through the relevant DOI and the license). See: https://creativecommons.org/licenses/by-nc-nd/4.0/.

\section{References}

1. Mina R, Brunner HI. Pediatric lupus--are there differences in presentation, genetics, response to therapy, and damage accrual compared with adult lupus? Rheum Dis Clin North Am 2010;36:53-80, vii-viii.

2. Watson L, Leone V, Pilkington C, et al. Disease activity, severity, and damage in the UK Juvenile-Onset Systemic Lupus Erythematosus Cohort. Arthritis Rheum 2012;64:2356-65.

3. Hiraki LT, Feldman CH, Liu J, et al. Prevalence, incidence, and demographics of systemic lupus erythematosus and lupus nephritis from 2000 to 2004 among children in the US Medicaid beneficiary population. Arthritis Rheum 2012;64:2669-76.

4. Sun L, Xu H, Liu HM, et al. Long-term follow-up of 101 cases with pediatric lupus nephritis in a single center in Shanghai. Zhonghua Er Ke Za Zhi 2011;49:819-24.

5. Hochberg MC. Updating the American College of Rheumatology revised criteria for the classification of systemic lupus erythematosus. Arthritis Rheum 1997;40:1725.

6. Smith EMD, Yin P, Jorgensen AL, et al. Clinical predictors of active LN development in children - evidence from the UK JSLE Cohort Study. Lupus 2018;27:2020-8.

7. Flenner E, Elgeti F, Luft FC, et al. The case I intractable diuretic resistance in a young woman. Kidney Int 2012;81:221-2.

8. Bertsias GK, Tektonidou M, Amoura Z, et al. Joint European League Against Rheumatism and European Renal Association-European Dialysis and Transplant Association (EULAR/ERA-EDTA) recommendations for the management of adult and paediatric lupus nephritis. Ann Rheum Dis 2012;71:1771-82.

9. Esdaile JM, Abrahamowicz M, Joseph L, et al. Laboratory tests as predictors of disease exacerbations in systemic lupus erythematosus. Why some tests fail. Arthritis Rheum 1996;39:370-8.

10. Bombardier C, Gladman DD, Urowitz MB, et al. Derivation of the SLEDAI. A disease activity index for lupus patients. The Committee on Prognosis Studies in SLE. Arthritis Rheum 1992;35:630-40.

11. National High Blood Pressure Education Program Working Group on High Blood Pressure in Children and Adolescents. The fourth report on the diagnosis, evaluation, and treatment of high blood pressure in children and adolescents. Pediatrics 2004;114:555-76.

12. Li F. Chinese Ophthalmology. Beijing: People's Medical Publishing House 2016; P1473, 1583, 1772.

13. Anthamatten A, Parish A. Clinical Update on Osteoporosis. J Midwifery Womens Health 
2019;64:265-75.

14. Balci S, Ekinci RMK, Bayazit AK, et al. Juvenile systemic lupus erythematosus: a single-center experience from southern Turkey. Clin Rheumatol 2019;38:1459-68.

15. Wan X, Wang W, Liu J, et al. Estimating the sample mean and standard deviation from the sample size, median, range and/or interquartile range. BMC Med Res Methodol 2014;14:135.

16. Takei S, Maeno N, Shigemori M, et al. Clinical features of Japanese children and adolescents with systemic lupus erythematosus: results of 1980-1994 survey. Acta Paediatr Jpn 1997;39:250-6.

17. Sahin S, Adrovic A, Barut K, et al. Juvenile systemic lupus erythematosus in Turkey: demographic, clinical and laboratory features with disease activity and outcome. Lupus 2018;27:514-9.

18. Al Arfaj AS, Khalil N. Clinical and immunological manifestations in 624 SLE patients in Saudi Arabia. Lupus 2009; 18:465-73.

19. Abdwani R, Rizvi SG, El-Nour I. Childhood systemic lupus erythematosus in Sultanate of Oman: demographics and clinical analysis. Lupus 2008;17:683-6.

20. Couture J, Silverman ED. Update on the pathogenesis and treatment of childhood-onset systemic lupus erythematosus. Curr Opin Rheumatol 2016;28:488-96.

21. Abdel-Hafez MA, Abdel-Nabi H. Juvenile systemic lupus erythematosus: onset patterns and short-term outcome in Egyptian children, a single-center experience. Lupus 2015;24:1455-61.

22. The American College of Rheumatology nomenclature and case definitions for neuropsychiatric lupus syndromes. Arthritis Rheum 1999;42:599-608.

23. Concannon A, Rudge S, Yan J, et al. The incidence, diagnostic clinical manifestations and severity of juvenile systemic lupus erythematosus in New Zealand Maori and Pacific Island children: the Starship experience (2000-

Cite this article as: Wei Q, Wang W, Dong Y, Zhong L, Song H. Five years follow-up of juvenile lupus nephritis: a single-center retrospective cohort study. Ann Palliat Med 2021;10(7):73517359. doi: 10.21037/apm-21-720
2010). Lupus 2013;22:1156-61.

24. Cabral M, Escobar C, Conde M, et al. Juvenile systemic lupus erythematosus in Portugal: clinical and immunological patterns of disease expression in a cohort of 56 patients. Acta Reumatol Port 2013;38:274-85.

25. Cavalcante EG, Aikawa NE, Lozano RG, et al. Chronic polyarthritis as the first manifestation of juvenile systemic lupus erythematosus patients. Lupus 2011;20:960-4.

26. Adelowo OO, Olaosebikan BH, Animashaun BA, et al. Juvenile systemic lupus erythematosus in Nigeria. Lupus 2017;26:329-33.

27. Zhang CX, Cai L, Zhou ZY, et al. Clinical manifestations, immunological features and prognosis of Chinese pediatric systemic lupus erythematosus: A single-center study. Int J Rheum Dis 2019;22:1070-6.

28. Alunno A, Padjen I, Fanouriakis A, et al. Pathogenic and Therapeutic Relevance of JAK/STAT Signaling in Systemic Lupus Erythematosus: Integration of Distinct Inflammatory Pathways and the Prospect of Their Inhibition with an Oral Agent. Cells 2019;8:898.

29. Ripoll È, de Ramon L, Draibe Bordignon J, et al. JAK3STAT pathway blocking benefits in experimental lupus nephritis. Arthritis Res Ther 2016;18:134.

30. Noël V, Lortholary O, Casassus P, et al. Risk factors and prognostic influence of infection in a single cohort of 87 adults with systemic lupus erythematosus. Ann Rheum Dis 2001;60:1141-4.

31. Ruiz-Irastorza G, Ramos-Casals M, Brito-Zeron P, et al. Clinical efficacy and side effects of antimalarials in systemic lupus erythematosus: a systematic review. Ann Rheum Dis 2010;69:20-8.

32. Chinese Rheumatism Data Center (CRDC) and CSTAR Co-authors. Expert consensus on hydroxychloroquine in the treatment of rheumatic diseases. Chin J Rheumatol 2014;18:148-50. 